\title{
In vitro Evaluation of PEGylated-Mucin Matrix as Carrier for Oral Delivery of Metformin Hydrochloride
}

\author{
MA Momoh ${ }^{1 *}$, MO Adedokun ${ }^{2}$, MU Adikwu ${ }^{1}$ and CE Ibezim ${ }^{1}$ \\ ${ }^{1}$ Drug Delivery Research Unit, Department of Pharmaceutics, University of Nigeria, Nsukka 410001, ${ }^{2}$ Department of Pharm. \\ Tech and Pharmaceutical Microbiology, University of Uyo, Uyo, Nigeria \\ *For correspondence: Email: jointmomoh@yahoo.com; Tel: 234-8037784357
}

\begin{abstract}
Purpose: To formulate metformin hydrochloride-loaded PEGylated-mucin microparticles and evaluate their in vitro properties.

Method: Three different formulations of metformin hydrochloride (MTH) (PEG-M1, PEG-M2 and PEGM3) were prepared using PEGylation method. PEG-8000 and snail mucin, in a ratio of 1:3, were PEGylated together using solvent interaction principle. Loading of MTH into the matrix was by diffusion method and the microparticles characterized for particle size, zeta potential, polydispersity index, stability and in vitro release in phosphate buffer ( $\mathrm{pH}$ 7.4).

Results: Maximum yield and encapsulation were 97 and $87 \%$ respectively. Zeta potential was -37.7, 42.3 and $-46.2 \mathrm{mV}$ for PEG-M1, PEG-M2 and PEG-M3 with a corresponding polydispersity index (PDI) of $0.320,0.374$ and 0.398 , respectively. Particles size was 85, 115, and $145 \mu \mathrm{m}$ for PEG-M1, PEG-M2 and PEG-M3, respectively, and they showed a unimodal distribution. Drug release was biphasic and exhibited controlled release pattern with maximum release of $92 \%$ in $18 \mathrm{~h}$ compared to $81 \%$ in $6 \mathrm{~h}$ for the conventional formulation.

Conclusion: Extended release metformin hydrochloride formulations were successfully developed using PEGylated mucin matrices.
\end{abstract}

Keywords: Drug delivery, Extended release, Polyethylene glycol, Mucin PEGylation, Encapsulation, Zeta potential, Polydispersity index

Tropical Journal of Pharmaceutical Research is indexed by Science Citation Index (SciSearch), Scopus, International Pharmaceutical Abstract, Chemical Abstracts, Embase, Index Copernicus, EBSCO, African Index Medicus, JournalSeek, Journal Citation Reports/Science Edition, Directory of Open Access Journals (DOAJ), African Journal Online, Bioline International, Open-J-Gate and Pharmacy Abstracts

\section{INTRODUCTION}

Modification of drug carrier has been justified and recently received increased attention as a means to create new entities that perform better than the native materials $[1,2]$ in terms of improved drug targeting, half-life, and bioavailability [3]. Several techniques have been explored in carrier modifications [3]. Apart from hybrid polymers with superior functional and physicochemical properties; PEGylation has also been proved to have superior characteristics to hybrid polymers. PEGylation is one of the most promising and extensively studied strategies for improving the pharmacokinetics and pharmacodynamics of drugs [4]. PEGylation is simply defined as the addition of polyethylene glycol (PEG) to a functional group or into a molecule.

Various PEGylation techniques have been used to enhance the delivery of both hydrophilic and lipophilic drugs. PEGylation has been shown to increase therapeutic efficacy by enabling increased drug concentration, improved biodistribution and longer dwelling time at the site of action [4]. Furthermore, the widening 
availability of PEGs with specific characteristics offers flexibility of application with respect to improving the bioavailabilty of both lipophilic and hydrophilic drugs and manipulating their release profile [5]. The proven safety of PEGs and efficacy of PEGylated-based carriers make them potential alternative drug carrier materials that have gained tremendous support in the pharmaceutical industries [6].

Metformin is an antidiabetic drug with pronounced blood glucose lowering effect. However, poor bioavailability and high intake of the drug are big challenges to the clinician. The study was aimed at evaluating metformin-loaded PEGylayed-mucin (PEG-8000 and mucin) both in vitro and in vivo in order to improve oral bioavailability of the dug and reduce its dosing frequency.

Mucins are high-molecular weight glycoproteins found on the surface of epithelial tissues $[7,8]$. In the gastrointestinal tract, mucin protects the mucus membrane against the harsh conditions of the gastric environment caused by hydrochloric acid. It also controls the diffusion of molecules across the mucus membrane. The functional properties of mucin as a protective coat make it a potential pharmaceutical excipient for new drug delivery concepts.

\section{EXPERIMENTAL}

\section{Materials}

Polyethylene glycol-8000 (Sigma St. Lious, USA), citric acid, sodium hydroxide (Merck, Germany), sodium chloride, acetone, concentrated $\mathrm{HCl}$ (BDH, England). Phosphate buffer $\mathrm{pH} 7.4$ (PBS) were prepared following the compendium (USP XXIII) specification. Metformin hydrochloride was a gift from (Farmex Meyer Pharma Ltd, Ikeja, Lagos, Nigeria). All other reagents and solvents were analytical grade and were used as supplied. Distilled water was obtained from a glass still.

\section{Extraction and purification of mucin from snail}

The method described [9], was employed for the extraction of mucin. The giant African land snails (Archachatina marginata) used was procured from a local market in Nsukka, Enugu State. The shells of the giant African land snails were knocked open at the apex and a spirally coiled rod was inserted to remove the fleshy body. The fleshy parts were then placed in $250 \mathrm{ml}$ of water and washed several times until the (slime) mucin was completely washed off. These washings were pooled together in a plastic bucket, precipitated with chilled acetone. To obtain the soluble mucin, the precipitate obtained was further dissolved in distilled water and homogenized for $2 \mathrm{~h}$ at $4{ }^{\circ} \mathrm{C}$ and thereafter exhaustively dialysed against distilled water using a $12 \mathrm{KDa}$ molecular weight cut-off (MWCO) dialysis membrane. The dialysate was finally centrifuged at $8,000 \mathrm{rpm}$ for $1 \mathrm{~h}$ to yield a supernatant of water-soluble mucus glycoprotein layers and lower layer of insoluble mucus glycoprotein. The supernatants were collected separately, pooled and lyophilized at $-40{ }^{\circ} \mathrm{C}$ for $48 \mathrm{~h}$ to obtain flakes of soluble (S) mucin, which were powdered and used for the study.

\section{Preparation of PEGylated-mucin}

A known quantity of PEG 8000 (5 g) was dispersed in $250 \mathrm{ml}$ of water and $15 \mathrm{~g}$ quantity of soluble-mucin was similarly weighed out dispersed in another beaker contained $250 \mathrm{ml}$ of water, and allowed to stand for $48 \mathrm{~h}$, thereafter the two container were mixed together and further allowed to stand for $48 \mathrm{~h}$ to give a ratio of 1:3 of PEG: Mucin. The mixture was left to stand for another $24 \mathrm{~h}$ in order to attain maximum hydration and possible bond interaction due to the functional groups of mucin. The mixture was then precipitated using chilled acetone, the precipitate was harvested and dried and kept for further used. The choice of the different quantities of PEG and the mucin was informed by the result of the earlier study of bioadhesive systems [10].

\section{Loading of metformin hydrochloride into PEGylated-mucin matrix}

Metformin $\mathrm{HCl}$ was loaded into the various PEGylated-microparticles by diffusion method [11]. Briefly, $2 \mathrm{~g}$ of the matrix produced was placed in a flat Petri-dish plate, $250 \mathrm{mg}$ quantities of the MTH was dispersed in $2 \mathrm{ml}$ distilled water and allowed to dispersed, this was then introduced into the matrix in petri-dish and gentle stirred and was allow to stand for $5 \mathrm{~h}$ at $10{ }^{\circ} \mathrm{C}$, we work at this temperature because of the sensitive nature of mucin. After which the hydrated microparticles mixed with the drug were then freeze-dried for $12 \mathrm{~h}$, and MTH-loadedmatrix was obtained. By adding increasing concentrations of MTH (250, 500 and $1000 \mathrm{mg})$ to the PEGylated-matrix following the procedure indicated above, MTH-loaded PEGylated-matrix were obtained. The resulting formulations were dried and labeled as PEG-M1, PEG-M2 and PEG-M3 for 250, 500 and $1000 \mathrm{mg}$ of MTH, respectively. 


\section{Morphological studies}

Morphology of the formulations was examined. Briefly, $20 \mathrm{mg}$ of the MPs from each batch was dispersed in distilled water and smeared on a microscopic slide using a glass rod. The mixture was covered with a cover slip and viewed with a digital light microscope (Leica Diestar, Germany) with an image captured Moticam camera at a magnification x 1000 .

Submicron particle size analysis of the PEGylated microparticles was performed using a Zetasizer (ZEN 3600, Malvern Instruments, UK). Measurements were made at $25{ }^{\circ} \mathrm{C}$ at a scattering angle of $90^{\circ}$. The mean particle size and polydispersity index were determined in a single run while the zeta potential was similarly determined by phase analysis light scattering (PALS) using also a Zetasizer.

\section{Entrapment efficiency determination}

Accurately weighed, $100 \mathrm{mg}$ of MTH-loaded PEGylated-mucin matrices were taken and crushed in pestle and mortar. The crushed powders were placed in $250 \mathrm{ml}$ of phosphate buffer ( $\mathrm{pH} 7.4)$, and kept for $2 \mathrm{~h}$ with occasionally shaking at $37 \pm 0.5^{\circ} \mathrm{C}$. The solution was filtered through Whatman® filter paper (no. 20) and drug content in the filtrate was determined using a UVvisible spectrophotometer (UNICO-3102, England). Drug entrapment efficiency (EE) of $\mathrm{MTH}$ was calculated using Eq 1.

$\mathrm{EE}(\%)=(\mathrm{Am} / \mathrm{At}) 100$

where $A m=$ actual drug loading and At is theoretical drug loading.

\section{Drug release studies}

The dissolution medium consisted of $250 \mathrm{~mL}$ of freshly prepared phosphate buffer ( $\mathrm{pH}$ 7.4) maintained at $37.2{ }^{\circ} \mathrm{C}$. The polycarbonate dialysis membrane used was pre-treated by soaking it in the dissolution medium for $24 \mathrm{~h}$ prior to the commencement of each release experiment. In each case, $0.5 \mathrm{~g}$ of the formulated MPs was placed in the dialysis membrane containing $5 \mathrm{~mL}$ of the dissolution medium, securely tied with a thermo-resistant thread and then immersed in the dissolution medium under agitation provided by the paddle at $100 \mathrm{rpm}$. At predetermined time intervals, $5 \mathrm{~mL}$ portions of the dissolution medium were withdrawn, filtered and analyzed spectrophotometrically (Jenway 6042, Germany) at $242 \mathrm{~nm}$. For each sample withdrawn, an equivalent volume $(5 \mathrm{~mL})$ of phosphate buffer maintained at the same temperature was added to the contents of the dissolution medium to maintain sink conditions throughout the release period. The amount of drug released at each time interval was determined with reference to the standard Beer's plot for metformin in phosphate buffer.

\section{Stability study}

Stability evaluation is key to the pharmaceutical formulator, in this study, we evaluate the stability of our formulation to ascertain the protective action of the carrier. Batch (PEG-M3) of the formulation was stored at $4{ }^{\circ} \mathrm{C}$, the selection of this batch was based on the in vitro release studied, also the high content of the drug incorporated in the formulation make it a good candidate for the stability evaluation. Samples were taken after 3 and 6 months period of storage and the percentage of MTH retained in the formulation as well as the changes in particles size, PDI, EE (\%), ZP and in vitro release were evaluated.

\section{Statistical analysis}

All experiments were performed in replicates for validity of statistical analysis. Results were expressed as mean \pm SD. ANOVA and Student's t-test were performed on the data sets generated using SPSS (Version 17, SPSS Inc, New York, USA). Differences were considered significant at $p<0.05$.

\section{RESULTS}

The recovery rate of each of the formulation was high indicating that the method use in this study was economical and can be scaled up for commercial use. More so, the percentage recovery across the batches increased with increased drug loading showing that the formulation technique adopted was reliable. The $\%$ recovered are; 89.8, 96.2 and 97.9 for PEGM1, PEG-M2 and PEG-M3, respectively. The encapsulation efficiency (EE \%) were 88, 90 and $97 \%$ for PEG-M1, PEG-M2 and PEG-M3 respectively, indicate that drug encapsulation efficiency increased with increase in the concentration of MTH.

The particle size of the MTH loaded-PEGylated mucin, as measured by photon correlation spectroscopy, was $85.5,109.3$ and $124.8 \mu \mathrm{m}$ for formulations PEG-M1, PEG-M2 and PEG-M3 respectively with a corresponding polydispersity index (PDI) of $0.320,0.374$ and 0.398 . The zeta potential for all the formulations were negative and were as follows; $-37.7,-42.3$ and $-46.2 \mathrm{mV}$ respectively. The distribution here can be said to 
A

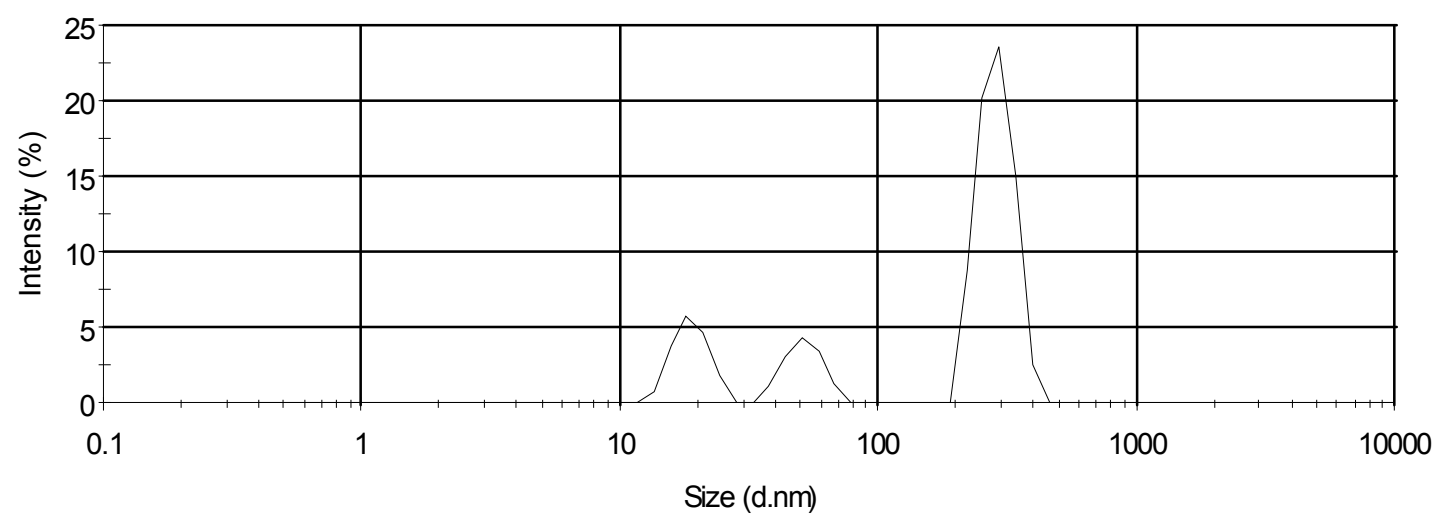

B

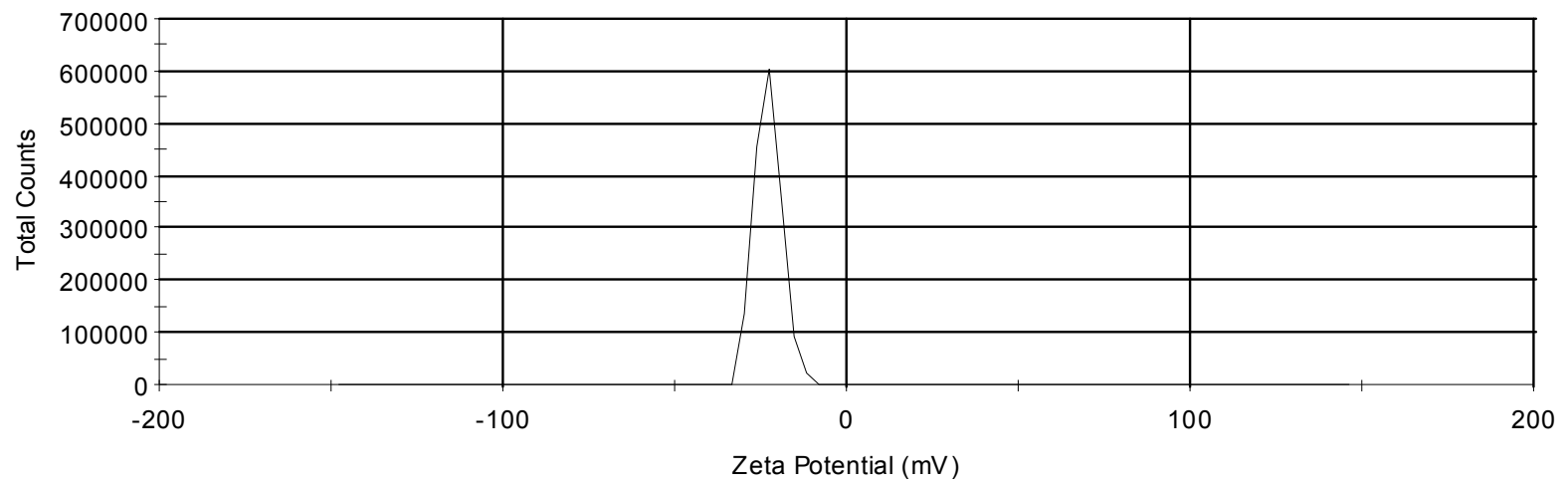

Fig 1: Particle size distribution $(A)$ and zeta potential $(B)$ of the formulations

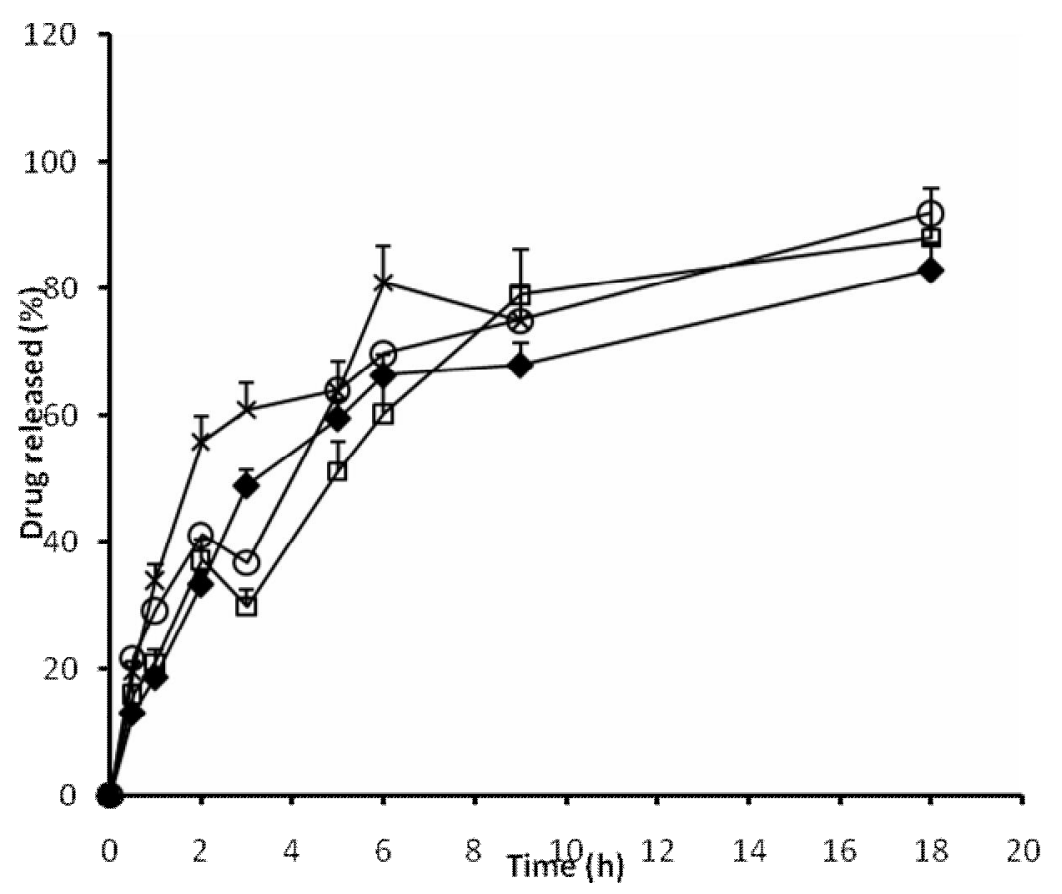

Fig. 2: Release profile of metformin hydrochloride from the PEGylated-mucin matrix; (•) = PEG-M1, $(\square)=$ PEGM2, $(\Delta)=$ PEG-M3, $(x)=$ Mkt. Note: PEG-M1 = batch loaded with $250 \mathrm{mg}$, PEG-M2 = loaded with $500 \mathrm{mg}$, PEG-M3 = loaded with $1000 \mathrm{mg}$ of metformin hydrochloride and Mkt = marketed sample of the drug 
Table 1: Characteristics of batch stored at $\left(4^{\circ} \mathrm{C}\right)$ vs freshly formulated batch (PEG-M3)

\begin{tabular}{lccccc}
\hline PEG-M3 & Particle size $(\boldsymbol{\mu m})$ & Drug content (\%) & PDI & EE (\%) & ZP (-Mv) \\
\hline Freshly formulated (48 h) & $124.80 \pm 0.10$ & $94.30 \pm 0.15$ & 0.398 & $97.90 \pm 0.10$ & -46.2 \\
After storage (3 months) & $125.60 \pm 0.11$ & $91.40 \pm 0.21$ & 0.422 & $95.40 \pm 0.11$ & -49.3 \\
After storage (6 months) & $127.10 \pm 0.20$ & $89.60 \pm 0.13$ & 0.431 & $94.20 \pm 0.22$ & -52.8 \\
\hline
\end{tabular}

be unimodal in all the formulations. The PDI also depicting that the formulation is relatively stable and can be effectively protect the incorporated drug, as shown in Fig 1.

The results of the in vitro release studies of MTH are shown in Fig 2. The release rate of MTH from the preparations was dependent on the amount of the drug incorporated into the carrier. Maximum release of $92 \%$ was observed in PEGM3 which contained the highest amount of the drug in $18 \mathrm{~h}$, this was followed by PEG-M2 (88 $\%)$ and $83 \%$ for PEG-M1.

In all cases, the release was very good and show controlled release pattern when compared to the marketed sample (control) that showed a maximum release of $81 \%$ in $6 \mathrm{~h}$ and continue to decline after $8 \mathrm{~h}$ of the studied.

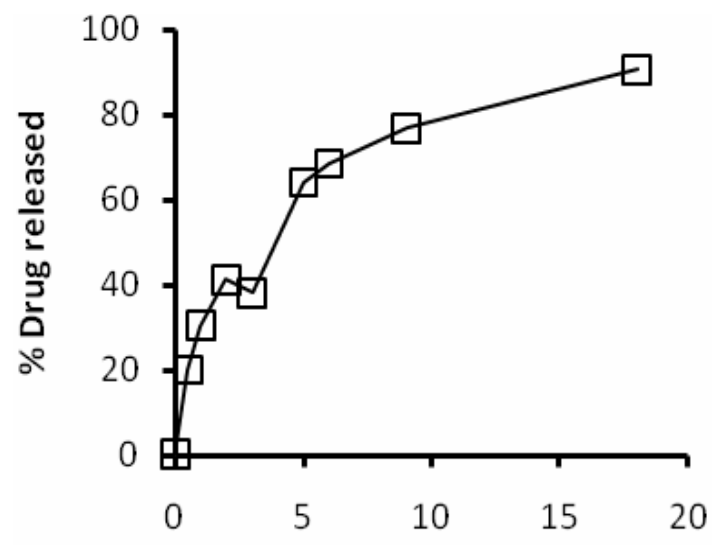

Time (h)

Fig 3: Release profile of metformin hydrochloride from the PEGylated-mucin matrix stored at $4{ }^{\circ} \mathrm{C},(\square)=$ PEG-M3 loaded with 1000 mg of MTH

The stability evaluation (Table 1) showed no significant change in the parameters evaluated after drug-loaded PEGylated-mucin matrix were subjected stressed conditions. In vitro release studies showed that stored PEG-M3 had the same release ability as that of newly prepared PEG-M3 (Fig 3). The release values of the stored PEG-M3 were almost the same as those of the freshly prepared PEG-M3, indicating the PEGylated-mucin maintained their physical characteristics as a controlled release formulation. These results demonstrate that the PEG-M3 had good stability at $4{ }^{\circ} \mathrm{C}$.

\section{DISCUSSION}

PEGylation technology is one of the important drug delivery principles, which improves the absorption of poorly absorbable drugs. This carrier modification system for the delivery of active pharmaceutical agents are regarded as very safe and tend to modify the release of the incorporated drug, however, PEGylation that involves biological substance like mucin which is known to be a component of the GIT mucous system will not only protect the drug and stabilized formulations. They are also known to enhance the water solubility of hydrophobic and hydrophilic compounds and attain their better absorption into the biological system. Recent clinical studies have suggested however that PEGylation provides significant unexpected, and as yet unexplained, therapeutic benefits for protein and non protein-based medicines [3]. The most dramatic of these is the eradication of Hepatitis C virus in more than $50 \%$ of the patients who have been treated with PEGylated interferon $\alpha-2$ and ribavirin for 6 months [12]. Also, the therapeutics activities of some antibiotics have also improved [6].

The choice of snail mucin used in this study rather than the commercially purified one is as a result of a previous finding that the locally purified mucin resulted in a more accurate model system because protease treatment tended to degrade the commercially available and the glycoproteins causing a substantial difference in their bioadhesive properties [13].

Photomicrographs (not shown) indicate that the microparticles have a spherical shape and a fairly smooth surface and this was not dependent on the amount of drug incorporated into the formulation.. The morphology of the PEGylatedmucin indicated intimate interaction through molecular bonding, resulting from the interplay of the functional groups of the polymer chain; these interactions could have resulted from hydrogen and ionic bonding as well as from physical entanglement between the different polymer chain networks. Mucin is said to have well- 
defined networks that interact with the PEG functional groups which resulted in the creation of legend space for drug encapsulation.

Microparticles size was in the range of 85.5 $124.8 \mu \mathrm{m}$ the size was within the size that can be easily absorbed, it also showed a unimodal size distribution which was also a pointer toward good particles size formulation for oral drug delivery. The difference in particles sizes, though not statistically significant $(p>0.05)$, was due to the quantity of drug incorporated into the formulation. The particle formation process was very efficient, with recovery rate above $96 \%$ for the three batches.

Furthermore, PEGylated-mucin was able to encapsulate high amounts of the MTH (> $95 \%$ ), an indication that, this method could be utilize for commercial purpose. The zeta potential was negative, indicating that the loading of the MTH in the carrier did not change the surface charge. Higher zeta potential is an indication of the repulsive forces that are present and can be used to predict the stability of the formulation [13]. Particles of higher zeta potential with negative or positive charges tend to repel each other and have no tendency to aggregate or flocculate which would have affected the release of the incorporated drug.

The dividing line between stable and unstable suspension is generally taken as ZP values from +30 to $-30 \mathrm{mV}$ as stable (flocculated) and ZP values outside this range as unstable (deflocculated) [13]. Although batch $C$ had highest absolute zeta potential value (highest stability), other properties associated with this PEGylated-mucin carrier would preclude its use in drug delivery or carrier. The overall negative zeta potential of all the formulations would also contribute to stability of the microparticles when dispersed in the fluid medium, and provide surfaces for attachment of drugs, hence the high encapsulation efficiency recorded in this study [14].

The rapid release in the first $30 \mathrm{~min}$ is possibly due to a 'burst' effect caused by the leaching out of the unentrapped drug adhering to the surface of the PEGylated-mucin at the initiation of the release study. The control release of $\mathrm{MTH}$ from the formulation in SIF, in addition to the burst effect, may also be as a result of the hydrophilic nature of both carriers (PEG and mucin). There was a gradual slow release of the MTH after $6 \mathrm{~h}$ of the study; this could be attributed to the interconnectivity of the polymer complexes preventing the migration of the drug to the surface into the dissolution medium.

All the formulations showed between 82 and 92 $\%$ release in $18 \mathrm{~h}$. The biphasic release noticed is an advantage in oral drug formulation, as such initial release can be considered useful in the management of disease conditions that need a loading dose at the unset of treatment while the second phase serves as maintenance dose. Moreover, no significant difference was found among the three batches of the formulations, but when the release data were compared to the marketed formulation, there was a significant difference $(p<0.05)$. This indicates that PEGylation of PEG: mucin had some effect on the MTH release from the microparticles, which was a key in this study. All the batches of the formulations showed a steady and prolonged release in the buffer solution as compared to marketed sample. This was due to the ability of the high mucin content in the carrier to maintain their structural integrity by forming a stable gel network due to its high viscoelastic consistency and prevent drug expulsion [15].

However, PEG been a non-ionic surfactant used in this study may promotes steric repulsion of the particles and as a consequence dominates the attraction force, thereby culminating in enhanced product stability as demonstrated in the stability studied. This is so because of the hydrophilic moiety of polyethylene glycol chains (PEG) which shield the core of the microparticles isolating them from their aqueous surroundings and preventing the efflux of the drug to the surfaces. The net effect of this shielding action is the observed sustained release of the incorporated MTH in the microparticles studied. The in vitro findings are in agreement with those of found in in vivo studies where the (PEG) hydrophilic layer has been implicated in the slow plasma clearance of drug from the system $[15,16]$.

The stability study showed that a high proportion of the drug was retained, there was no significant change in the values of parameters such as particles size, PDI and ZP, showing that the products maintained their stability profile.

The low level of drug leakage can be ascribed to the role of mucin network in the retardation or expulsion of the drug from the formulation. In other words, the inter-polymer complexation between PEG and the mucin acts as a stabilizer; the inter-polymer complexation is a thermodynamically stable thereby forming a 
network chain that prevents the rapid expulsion of the incorporated drug.

The choice of the storage conditions was based on the nature of mucin since it can be easily denatured at high temperature or at a very low humidity, and also can absorb water leading to loss of its natural integrity. PEG also absorb water and can easily release its content due to it hydrophilic nature.

\section{CONCLUSION}

PEGylated mucin-loaded MTH obtained by diffusion method has good sustained release characteristics and stability, and would be suitable for oral delivery of metformin hydrochloride.

\section{REFERENCES}

1. Friedrich I, Müller-Goymann CC. Characterization of solidified reverse micellar solutions (SRMS) and production development of SRMS-based nanosuspensions, Eur J Pharm. Biopharm 2003; 56 . 111-199.

2. Rossi S, Sandri G, Ferrari F, Bonferoni MC, Caramells C. Development of films and matrices based on chitosan and polyacrylic acid for vaginal delivery of acyclovir. STP Pharma Sciences 2003; 13: 183-198.

3. Vila A, Gill H, McCallion O, Alonso MJ. Transport of PLA$P E G$ particles across the nasal mucosa: effect of particle size and PEG coating density, J. Control Rel 2004; 98: 231-244.

4. Li L, Liu J, Lin S, Liu E, Release of theophylline from polymer blend hydrogels, Int J Pharm. 2005; 298: 117-125

5. Mortazavi SA, Carpenter BG, Smart JD. Comparative study on the role played by mucus glycoprotein in the rheological behaviors of the mucoadhesive/mucosal interaction, Int J Pharm, 1992; 94: 195-201.

6. Ofokansi KC, Adikwu MU. Formulation and Evaluation of Microspheres Based on Gelatin-Mucin Admixtures for the Rectal Delivery of Cefuroxime Sodium. Trop J Pharm Res, 2007; 6 (4): 825-832.

7. Builders PF, Kunle OO, Adikwu MU. Preparation and characterization of mucinated agarose: a mucinagarose physical crosslink, Int. J. Pharm, 2008; 356: 174-180.

8. Adikwu MU, Alozie BU. Application of snail mucin dispersed in detarium gum gel in wound healing. Sci Res Essay, 2007; 2: 195-198.

9. Adikwu MU, Aneke KO, Builders PF. Biophysical properties of mucin and its use as a mucoadhesive agent in drug delivery: current development and future concepts, Nig J Pharm Res. 2005; 4: 60-69.

10. Momoh MA, Adikwu MU, Ibezim EC. Formulation and evaluation the bioadhesive properties of drug delivery system based on PEGylated mucin matrices. Asian Pac J Trop Med, 2010; 2: 461-464.

11. Momoh MA, Adikwu MU. Evaluation of oral insulin delivery using bovine mucin in non-diabetic rats. $J$ Pharm Res 2008; 7: 5-9.

12. Hadziyannis S. Peginterferon-alpha2a and ribavirin combination therapy in chronic hepatitis C: a randomized study of treatment duration and ribavirin dose. Ann Intern Med 2004; 140: 346- 355.

13. Kocevar-Nared J, Kristl J, Smid-Korbar AC. Comparative rheological investigation of crude gastric mucin and natural gastric mucus. J Biomaterials 1997; 18: 677681.

14. Zetasizer Nano Series, Malvern Instruments England, User Manual Issue 2.2, 2005.

15. Adikwu MU. Mucins and their potentials (editorial). Trop J Pharm Res 2006; 5: 581-582.

16. Rowe RC, Sheskey PJ, Owen SC. Handbook of Pharmaceutical Excipients, 5th edn, London: Pharmaceutical Press, 2006; $p 112$. 